\title{
Constitutive MHC class I molecules negatively regulate TLR-triggered inflammatory responses via the Fps-SHP-2 pathway
}

\author{
Sheng $\mathrm{Xu}^{1{ }^{*}+}$, Jin Hou ${ }^{1 \dagger}$, Xingguang Liu ${ }^{1}$, Chaofeng Han ${ }^{1}$, Peng Zhang ${ }^{1}$, Xuetao Cao ${ }^{1,2}$ \\ From 7th European Workshop on Immune-Mediated Inflammatory Diseases \\ Noordwijk aan Zee, the Netherlands. 28-30 November 2012
}

Toll-like receptors (TLRs) are key pattern-recognition receptors used by cells of the innate immune system to detect conserved components of pathogens, and have critical roles in host defense against microbial pathogens. However, the molecular mechanisms that fine-tune TLRtriggered innate responses remain to be fully elucidated. Previous studies suggested that major histocompatibility complex (MHC) molecules can mediate reverse signaling and have nonclassical functions[1,2]. The aggregation of MHC class I on cell surface activates signal pathways in $\mathrm{T}$ cells, B cells, tumor cells or endothelial cells and elicits various biological effects, such as cell apoptosis, activation or proliferation [3,4]. Crosslinkage of MHC class I on human NK cells induces intracellular tyrosine phosphorylation and inhibits NK cell cytotoxicity[5]. Here we found that constitutively expressed membrane MHC class I molecules attenuated TLR-triggered innate inflammatory responses via reverse signaling. TLR ligands triggered more inflammatory cytokines production in MHC Ideficient mice and macrophages. And MHC I-deficient mice were more susceptive to both endotoxin sepsis and E. coli infection, but more resistant to Listeria monocytogenes. The intracellular domain of MHC class I molecules was phosphorylated by the kinase Src after TLR activation, then the tyrosine kinase Fps was recruited via its $\mathrm{Src}$ homology 2 domain to phosphorylated MHC class I molecules. This led to enhanced Fps activity and recruitment of the phosphatase SHP-2, which interact with TRAF6 and inhibit its ubiqutination, and finally resulted in suppressed TLR-triggered innate inflammatory responses. We further found that MHC class I molecules on B cells could also attenuate BCR signaling by suppressing Lyn activation. So, constitutive MHC class I molecules may be not only antigen presenting molecules, but also involved in fine-tune of both innate and adaptive immune responses.

\section{Author details}

'National Key Laboratory of Medical Immunology \& Institute of Immunology, Second Military Medical University, Shanghai, China. ${ }^{2}$ National Key Laboratory of Molecular Biology, Chinese Academy of Medical Sciences, Beijing, China.

\section{Published: 28 November 2012}

\section{References}

1. Arosa FA, Santos SG, Powis SJ: Open conformers: the hidden face of MHC-I molecules. Trends Immunol 2007, 28(3):115-123.

2. Pedersen AE, Skov S, Bregenholt S, Ruhwald M, Claesson MH: Signal transduction by the major histocompatibility complex class I molecule. APMIS 1999, 107(10):887-895.

3. Yang J, Qian J, Wezeman M, et al: Targeting beta2-microglobulin for induction of tumor apoptosis in human hematological malignancies. Cancer Cell 2006, 10(4):295-307.

4. Zhang X, Rozengurt E, Reed EF: HLA class I molecules partner with integrin beta4 to stimulate endothelial cell proliferation and migration. Sci Signal 2010, 3(149):ra85.

5. Rubio G, Ferez X, Sanchez-Campillo M, et al: Cross-linking of MHC class I molecules on human NK cells inhibits NK cell function, segregates MHC I from the NK cell synapse, and induces intracellular phosphotyrosines. J Leukoc Biol 2004, 76(1):116-124.

doi:10.1186/1479-5876-10-S3-P7

Cite this article as: Xu et al: Constitutive MHC class I molecules negatively regulate TLR-triggered inflammatory responses via the Fps-SHP-2 pathway. Journal of Translational Medicine 2012 10(Suppl 3):P7.

\footnotetext{
† Contributed equally

'National Key Laboratory of Medical Immunology \& Institute of Immunology, Second Military Medical University, Shanghai, China

Full list of author information is available at the end of the article
}

(c) 2012 Xu et al; licensee BioMed Central Ltd. This is an Open Access article distributed under the terms of the Creative Commons Attribution License (http://creativecommons.org/licenses/by/2.0), which permits unrestricted use, distribution, and reproduction in any medium, provided the original work is properly cited. 